\title{
Coccidioidal meningitis with multiple aneurysms presenting with pseudo-subarachnoid hemorrhage: illustrative case
}

\author{
Rohin Singh, BS, Visish M. Srinivasan, MD, Joshua S. Catapano, MD, Joseph D. DiDomenico, MD, Jacob F. Baranoski, MD, and \\ Michael T. Lawton, MD
}

Department of Neurosurgery, Barrow Neurological Institute, St. Joseph's Hospital and Medical Center, Phoenix, Arizona

BACKGROUND Coccidioidomycosis is a primarily self-limiting fungal disease endemic to the western United States and South America. However, severe disseminated infection can occur. The authors report a severe case of coccidioidal meningitis that appeared to be a subarachnoid hemorrhage (SAH) on initial inspection.

OBSERVATIONS A man in his early 40s was diagnosed with coccidioidal pneumonia after presenting with pulmonary symptoms. After meningeal spread characterized by declining mental status and hydrocephalus, coccidioidal meningitis was diagnosed. The uniquely difficult aspect of this case was the deceptive appearance of SAH due to the presence of multiple aneurysms and blood draining from the patient's external ventricular drain.

LESSONS Coccidioidal infection likely led to the formation of multiple intracranial aneurysms in this patient. Although few reports exist of coccidioidal meningitis progressing to aneurysm formation, patients should be closely monitored for this complication because outcomes are poor. The presence of basal cistern hyperdensities from a coccidioidal infection mimicking SAH makes interpreting imaging difficult. Surgical management of SAH can be considered safe and viable, especially when the index of suspicion is high, such as in the presence of multiple aneurysms. Even if it is unclear whether aneurysmal rupture has occurred, prompt treatment is advisable.

https://thejns.org/doi/abs/10.3171/CASE21424

KEYWORDS aneurysm; coccidioidal meningitis; coccidioidomycosis; infectious aneurysms; pseudo-subarachnoid hemorrhage; subarachnoid hemorrhage

Coccidioidomycosis, a fungal disease caused by Coccidioides immitis and other related organisms, is endemic to the western United States and South America. ${ }^{1}$ The first case of coccidioidomycosis was described by Posadas in Argentina in $1892 .^{2}$ The patient, a 33-year-old cavalryman, noted what he thought to be a spider bite on his face. As the skin lesion worsened and spread across his body, he sought treatment from various hospitals across the country. He eventually received a diagnosis of mycosis fungoides infection; because no adequate treatments were available at the time, he died as a result of the infection after several years.

Coccidioidal infection is now commonly recognized, and its initial form is colloquially termed valley fever. ${ }^{3}$ Symptoms can range from fatigue, cough, and headaches to granulomatous skin lesions or chorioretinitis, depending on the site and severity of infection. ${ }^{4,5}$ Coccidioidomycosis is primarily a self-limiting disease that does not require intervention. However, severe cases of disseminated infection also occur., ${ }^{6,7}$ Coccidioidomycosis can spread via lymphohematogenous dissemination to various organ systems and cause a spectrum of disease presentations. The disease is common in the United States, specifically in southern Arizona, where it has a suspected incidence of $1 \%$ to $3 \%$, although most cases are asymptomatic. ${ }^{8}$ However, the incidence appears to be steadily increasing with population migration to the region, as indicated by the fourfold increase in cases between 1997 and $2006 .^{8}$

Coccidioidal meningitis $(\mathrm{CM})$ is the most devastating of the various manifestations of coccidioidomycosis and is associated with

ABBREVIATIONS $\mathrm{CM}=$ coccidioidal meningitis; $\mathrm{CNS}=$ central nervous system; $\mathrm{CT}=$ computed tomography; $\mathrm{EVD}=$ external ventricular drain; $\mathrm{MCA}=$ middle cerebral artery; $\mathrm{SAH}=$ subarachnoid hemorrhage.

INCLUDE WHEN CITING Published October 11, 2021; DOI: 10.3171/CASE21424

SUBMITTED July 23, 2021. ACCEPTED September 2, 2021.

(c) 2021 The authors, CC BY-NC-ND 4.0 (http://creativecommons.org/licenses/by-nc-nd/4.0/). 
high morbidity and mortality. In a 2016 review of 117 patients with CM, Stockamp and Thompson ${ }^{9}$ found that $91 \%$ died within 1 year of diagnosis and that all died within 2 years. The most severe sequelae of $\mathrm{CM}$ are hemorrhage, hydrocephalus, vasculitis, and ischemia. The incidence of $\mathrm{CM}$ is variable. Current estimates indicate approximately 200 to 300 cases of CM per year in the United States, although these rates are highly variable and will most likely increase as the overall rate of coccidioidal infections increases. ${ }^{10}$

In this report, we describe a unique case of coccidioidomycosis causing $\mathrm{CM}$ that appeared to be a subarachnoid hemorrhage (SAH) on initial inspection.

\section{Illustrative Case}

A man in his 40s with a history of end-stage renal disease and human immunodeficiency virus infection presented to the emergency department after 2 weeks of hiccups that progressed to bilateral chest pain which worsened with inspiration. He was receiving dialysis and antiretroviral therapy, although he had intermittent periods without care because of a lack of health insurance. He denied having fevers, chills, and fatigue, but he did have a nonproductive cough. Radiographic imaging of the chest revealed diffuse bilateral ground-glass opacities and a cavitary lung lesion (Fig. 1A). The patient was determined to have coccidioidal pneumonia. He was treated with fluconazole therapy and discharged upon stabilization of his symptoms.

His follow-up examination 1 month later revealed bibasilar-predominant consolidations superimposed on moderate pulmonary edema and effusions that indicated a chronic coccidioidal infection. The patient was treated with clonidine and advised to continue fluconazole treatment for the following year.
Four months later, the patient received care at another hospital, where he received a diagnosis of $\mathrm{CM}$; he developed agitation and diminished sensorium. He was intubated and transferred to our center. Computed tomography (CT) of the head revealed enlarged lateral, third, and fourth ventricles consistent with communicating hydrocephalus (Fig. 1B). CT angiography demonstrated multiple aneurysms: a small basilar apex aneurysm (Fig. 1C) and bilateral posterior communicating artery aneurysms and a left dorsal internal carotid artery aneurysm (Fig. 1D). An external ventricular drain (EVD) was placed to treat the hydrocephalus.

The following day, the patient had sudden drainage of bright red blood from the EVD, prompting concern for aneurysmal rupture. CT of the head demonstrated diffuse hyperdensities in the basal cisterns, obscuring visualization of possible aneurysmal SAH (Fig. 1E). However, CT scans also showed a clear right acute subdural hematoma with a midline shift (Fig. 2A). The patient was taken for emergent craniotomy. A right pterional craniotomy was performed, and the subdural hematoma was evacuated. Upon inspection, all aneurysms were intact, and the blood was determined to be of subdural origin, likely caused by the patient's CM (Fig. 2B and C). At this time, the treatment team decided that the patient would still benefit from aneurysmal clipping because his chronic infection increased the risk of rupture. Therefore, the aneurysms of the posterior communicating artery and the left dorsal internal carotid artery were clipped (Fig. 2D and E, Video 1). The patient tolerated the procedure well and regained some consciousness after the operation, opening his eyes and localizing. He continued to receive fluconazole therapy and received supportive care for his various other conditions. He was discharged to a skilled nursing facility 2 weeks after the operation.

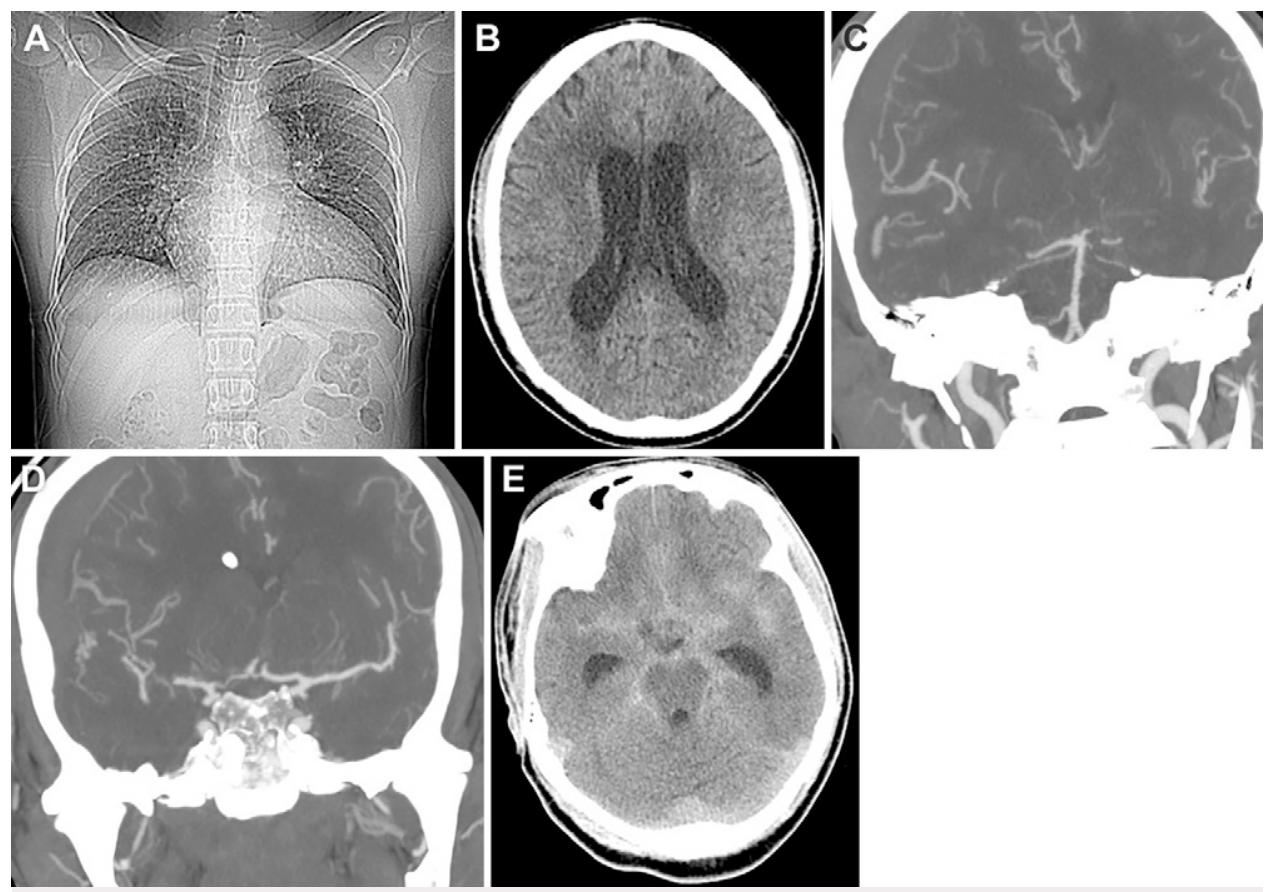

FIG. 1. Initial clinical imaging. A: Pulmonary radiograph displaying extensive ground-glass opacities. B: Axial CT before EVD placement showing dilated ventricles from hydrocephalus. C: Coronal CT angiogram showing basilar tip aneurysm. D: Coronal CT angiogram displaying posterior communicating artery and dorsal internal carotid artery aneurysms. E: Axial CT after EVD placement showing hyperdensity of basal cisterns. 

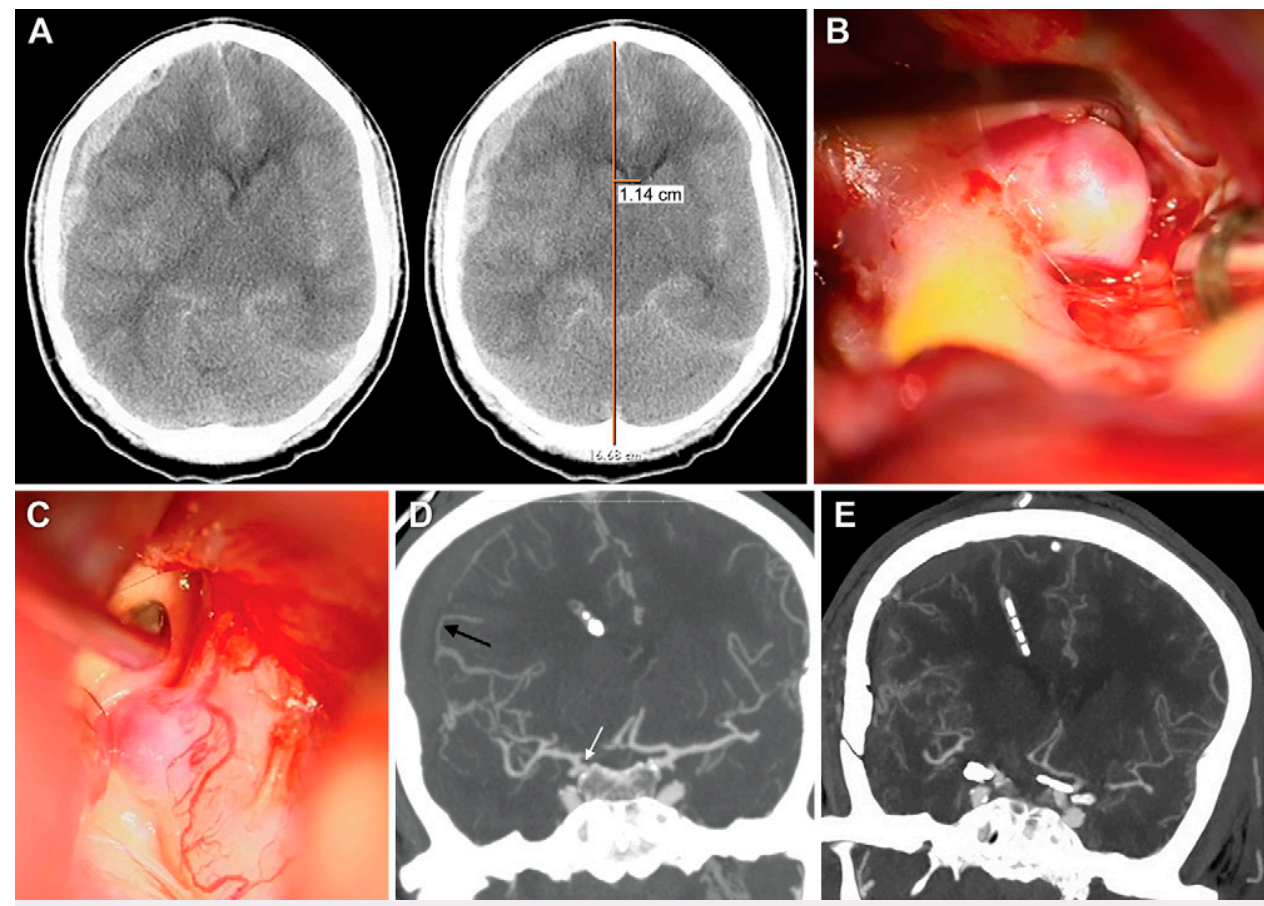

FIG. 2. A: Axial CT displaying acute subdural hemorrhage with 1.1-cm midline shift. B: Intraoperative photograph of the posterior communicating artery aneurysm with extensive inflammatory adhesions. C: Intraoperative photograph of the dorsal internal carotid artery aneurysm with extensive inflammatory adhesions. $\mathbf{D}$ and E: Postoperative coronal CT angiograms displaying clipped posterior communicating artery aneurysm (white arrow, D) and dorsal internal carotid artery aneurysm and the EVD in the right lateral ventricle (E). The subdural hemorrhage can be seen in $\mathbf{D}$ (black arrow). Figure 2B and $C$ are used with permission from Barrow Neurological Institute, Phoenix, AZ.

VIDEO 1. Operative video showing the inflammatory milieu of the suprasellar, chiasmatic, and carotid cisterns, with multiple aneurysms clipped through a transsylvian approach. Used with permission from Barrow Neurological Institute, Phoenix, AZ. Click here to view.

\section{Discussion}

\section{Observations}

This case was exceptionally complex because of the patient's unique presentation with the rare condition of CM. The patient's course followed the general timeline of initial coccidioidal pulmonary symptoms, with meningeal spread within a few months. In addition, his symptomatic presentation of fever, declining mental status, and hydrocephalus is commonly reported with $\mathrm{CM}$. What was unique about our patient and caused difficulty in treating him was the deceptive appearance of an SAH in the presence of multiple aneurysms and blood draining from his EVD.

Although what caused the formation of aneurysms in this patient is unknown, previous reports indicate that inflammatory states, such as infection and cancer, are associated with the development of aneurysms (Fig. 3). ${ }^{11-14} \mathrm{~A}$ similar report by Buchanan et al. ${ }^{15}$ illustrated the tragic case of a 26 -year-old man with persistent CM who rapidly developed multiple aneurysms. CT angiography of that patient demonstrated 10 different aneurysms and a true SAH predominantly filling the left sylvian fissure, which was treated microsurgically via a transsylvian approach and aneurysm trapping with bypass. Because the aneurysms were within the middle cerebral artery (MCA) territory, the surgeons performed an end-to-side superficial temporal-to-M2 MCA bypass, followed by trapping of the entire aneurysmal segment. ${ }^{16}$ Despite extensive medical and surgical management, the patient developed expressive aphasia, right facial droop, and left hemiparesis.

The pathophysiological mechanisms behind aneurysm formation may be shared by different infective organisms. A comparable report by Lee and $\mathrm{Kim}^{13}$ illustrated the rapid development of intracranial aneurysms in a patient with tuberculous meningitis. Their patient was a 34-year-old woman initially evaluated for headache and fever. The finding of CT of her brain was unremarkable, and she refused further medical work-up. She returned 6 days later with worsened symptoms and rightward gazeevoked nystagmus. Magnetic resonance imaging revealed leptomeningeal enhancement near the basal cisterns. She was admitted to the hospital, and, 2 days later, she developed tonic-clonic seizures and a sustained decrease in mental function. CT angiography at that time displayed an SAH with a basilar apex aneurysm that had not been evident 2 days earlier. Coil embolization was performed, but the patient did not improve and died within 1 month of admission. Our patient may have followed a similar disease course, with multiple aneurysms developing because of the chronic inflammatory state of his vasculature.

Further corroborating the theory that fungal infections are responsible for aneurysm formation are multiple reports of vasculitic complications arising from coccidioidal infection. Williams et al. ${ }^{17}$ reported on 10 patients with intracranial vasculitis caused by $\mathrm{CM}$. 


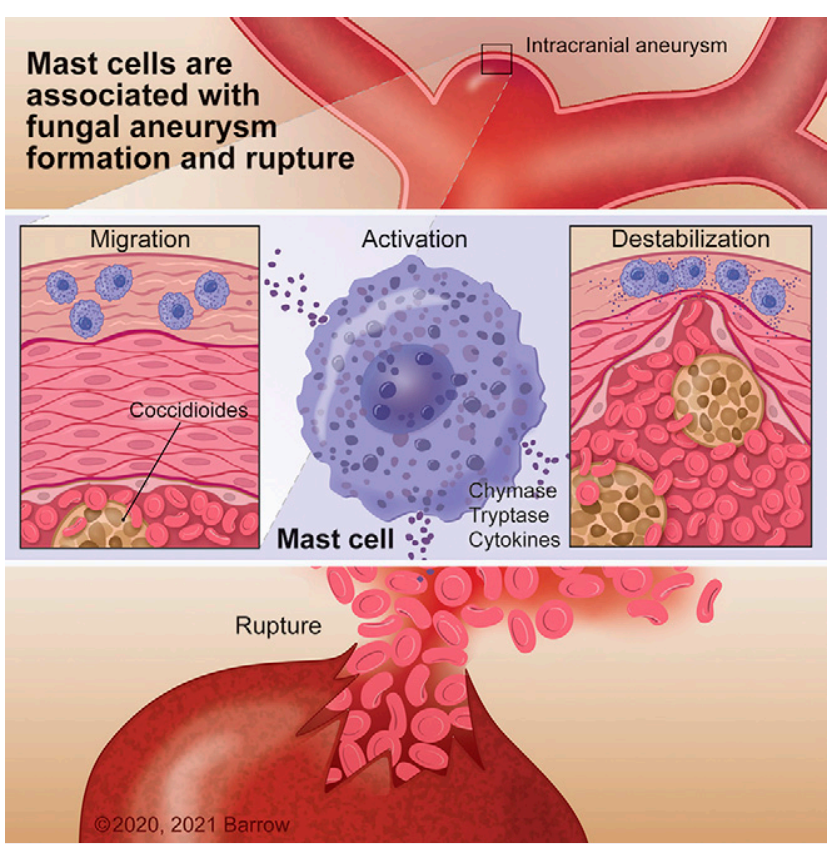

FIG. 3. Illustration of the association of mast cells with fungal aneurysm formation and rupture. Mast cells have been associated with aneurysm rupture via chymase, tryptase, and cytokine release, which leads to destabilization of aneurysm walls. Fungal aneurysms can form by inducing mast cell migration. Other inflammatory pathways, not pictured, may also play a role. ${ }^{14}$ Used with permission from Barrow Neurological Institute, Phoenix, AZ.

After their initial diagnosis, all 10 patients had rapid onset of central nervous system (CNS) symptoms, usually occurring within 3 to 4 weeks. Vasculitic complications were associated with high mortality rates, and seven patients died 5 weeks to 2.5 years after diagnosis. Furthermore, Erly et al. ${ }^{18}$ reported on two cases of disseminated coccidioidal infection that were complicated because of vasculitic pathology. One patient developed an aneurysm of the MCA, and the other displayed a granulomatous change and necrosis of the basilar artery. SAHs developed in both patients, with rapid progression to death. The high mortality rate associated with CNS infection necessitates early diagnosis to improve outcomes.

The inflammation and aneurysm formation in our patient may be partially attributable to mast cell activity. A report by Jiao et al. ${ }^{19}$ recognized the complex role that mast cells play in a variety of fungal infections. Mast cells were often found to facilitate the spread of the offending organism rather than to protect the host. In addition, numerous studies in mice have directly implicated mast cells in the development of aneurysm formation and rupture. ${ }^{14,20}$ A 2010 study by Ishibashi et al. ${ }^{20}$ found that mast cell degranulation had a direct role in the pathogenesis of aneurysm formation. Furukawa et al. ${ }^{14}$ induced aneurysm formation in adult mice and either stabilized (via cromolyn) or activated (via C48/80) mast cell activity. Mice with mast cell activation had significantly higher levels of aneurysm rupture. These studies suggest that mast cells may be a key mediator in fungal aneurysmal formation and rupture. Although future studies are needed to fully elucidate this relationship, treatment may include mast cell stabilization during routine management of fungal meningitis.

\section{Lessons}

Coccidioidal infection of the CNS is rare. Less than $1 \%$ of coccidioidomycosis cases result in hematogenous spread to other organ systems. $^{21}$ When coccidioidal infection spreads to the CNS, focal deficits such as gait disturbances, diplopia, and lethargy are common, especially if hydrocephalus occurs. ${ }^{22}$ Few reports exist of $\mathrm{CM}$ progressing to aneurysm formation, although patients should be closely monitored for this complication because outcomes are poor. ${ }^{15,23}$

Although our patient's disease progression resembles that in other published reports, the deceptive appearance of SAH caused by severe coccidioidal infection has never been described. The existence of pseudo-SAH from $\mathrm{CM}$ can make imaging interpretation more difficult, although it is advisable to treat this condition as a true SAH. Furthermore, in the setting of a confusing clinical pattern and the presence of multiple aneurysms, surgical management should be considered a safe and viable option. Careful consideration of the patient's risk factors and overall health must be taken into account before proceeding with surgical management versus other less invasive options. In the future, new investigational modalities such as vessel wall imaging may be an option for such patients. Either endovascular or microsurgical treatment may be considered, but the presence of multiple aneurysms in the setting of renal disease in our patient made microsurgical clipping more favorable.

\section{Acknowledgments}

We thank the staff of Neuroscience Publications at Barrow Neurological Institute for assistance with manuscript preparation.

\section{References}

1. American Academy of Family Physicians. What you should know about valley fever. Am Fam Physician. 2020;101(4):online.

2. Posadas A. A new case of mycosis fungoides with psorospermias. Article in Spanish. An Circ Med Argent. 1892;15:585-597.

3. Nelson R. Valley fever on the rise after years of decline in the USA. Lancet Infect Dis. 2019;19(11):1173.

4. Herrick KR, Trondle ME, Febles TT. Coccidioidomycosis (valley fever) in primary care. Am Fam Physician. 2020;101(4):221-228.

5. Jin J. Valley fever (coccidioidomycosis). JAMA. 2013;310(22):2470.

6. Gabe LM, Malo J, Knox KS. Diagnosis and management of coccidioidomycosis. Clin Chest Med. 2017;38(3):417-433.

7. Nordstrom B, Sherpa N, Marshall M, Chawla A, Heidari A, Johnson R. Coccidioidomycosis chorioretinitis. J Investig Med High Impact Case Rep. 2019;7:2324709619881561.

8. Johnson RH, Sharma R, Kuran R, Fong I, Heidari A. Coccidioidomycosis: a review. J Investig Med. 2021;69(2):316-323.

9. Stockamp NW, Thompson GR 3rd. Coccidioidomycosis. Infect Dis Clin North Am. 2016;30(1):229-246.

10. Williams PL. Coccidioidal meningitis. Ann N Y Acad Sci. 2007;1111: 377-384.

11. Eulate-Beramendi S, Alvarez-Vega MA, Gutierrez-Morales JC, Lopez-Garcia A. Meningioma associated with brain aneurysm: report of two cases. Turk Neurosurg. 2017;27(2):321-323.

12. Signorelli F, Sela S, Gesualdo L, et al. Hemodynamic stress, inflammation, and intracranial aneurysm development and rupture: a systematic review. World Neurosurg. 2018;115:234-244.

13. Lee $\mathrm{H}$, Kim T. Rapid development of intracranial aneurysm associated with tuberculous meningitis. Can J Neurol Sci. 2017;44(6): 730-731.

14. Furukawa $H$, Wada $K$, Tada $Y$, et al. Mast cell promotes the development of intracranial aneurysm rupture. Stroke. 2020;51(11): 3332-3339.

15. Buchanan IA, Ravina K, Strickland B, et al. Multiple intracranial aneurysms from coccidioidal meningitis: case report featuring 
aneurysm formation and spontaneous thrombosis with literature review. World Neurosurg. 2019;121:117-123.

16. Tayebi Meybodi A, Gadhiya A, Borba Moreira L, Lawton MT. Coding cerebral bypasses: a proposed nomenclature to better describe bypass constructs and revascularization techniques. J Neurosurg. Published online July 2, 2021. doi: https://doi.org/10.3171/2020.9. JNS202362.

17. Williams PL, Johnson R, Pappagianis D, et al. Vasculitic and encephalitic complications associated with Coccidioides immitis infection of the central nervous system in humans: report of 10 cases and review. Clin Infect Dis. 1992;14(3):673-682.

18. Erly WK, Labadie E, Williams PL, Lee DM, Carmody RF, Seeger JF. Disseminated coccidioidomycosis complicated by vasculitis: a cause of fatal subarachnoid hemorrhage in two cases. AJNR Am J Neuroradiol. 1999;20(9):1605-1608.

19. Jiao $Q$, Luo $Y$, Scheffel J, Zhao Z, Maurer M. The complex role of mast cells in fungal infections. Exp Dermatol. 2019;28(7): $749-755$.

20. Ishibashi R, Aoki T, Nishimura M, Hashimoto N, Miyamoto S. Contribution of mast cells to cerebral aneurysm formation. Curr Neurovasc Res. 2010;7(2):113-124.

21. Nasrawi F, Heidari A, Aljashamy T, et al. Disseminated coccidioidomycosis presenting as polyarticular septic arthritis: a case report. J Investig Med High Impact Case Rep. 2020;8:232470 9620974894.

22. Jackson NR, Blair JE, Ampel NM. Central nervous system infections due to coccidioidomycosis. J Fungi (Basel). 2019;5(3):54.
23. Hadley MN, Martin NA, Spetzler RF, Johnson PC. Multiple intracranial aneurysms due to Coccidioides immitis infection. Case report. J Neurosurg. 1987;66(3):453-456.

\section{Disclosures}

The authors report no conflict of interest concerning the materials or methods used in this study or the findings specified in this paper.

\section{Author Contributions}

Conception and design: Lawton, Singh. Acquisition of data: Singh, Srinivasan, DiDomenico, Baranoski. Analysis and interpretation of data: Lawton, Singh, Srinivasan, DiDomenico, Baranoski. Drafting the article: Lawton, Singh, Srinivasan, DiDomenico, Baranoski. Critically revising the article: all authors. Reviewed submitted version of manuscript: Lawton, Singh, Srinivasan, DiDomenico, Baranoski. Approved the final version of the manuscript on behalf of all authors: Lawton. Study supervision: Lawton.

\section{Supplemental Information \\ Video}

Video 1. https://vimeo.com/599581306.

\section{Correspondence}

Michael T. Lawton: c/o Neuroscience Publications, Barrow Neurological Institute, St. Joseph's Hospital and Medical Center, Phoenix, AZ. neuropub@barrowneuro.org. 